\title{
Promotion de projets par I'ISFM: nouvelle mise au concours
}

\author{
Werner Bauera, Raphael Stolzb ${ }^{b}$ Nadja Jennic \\ ${ }^{a}$ Dr, président de I'ISFM; ' ${ }^{2}$ r, vice-président de I'ISFM; ${ }^{\circ} M S c$, collaboratrice scientifique FMH/ISFM
}

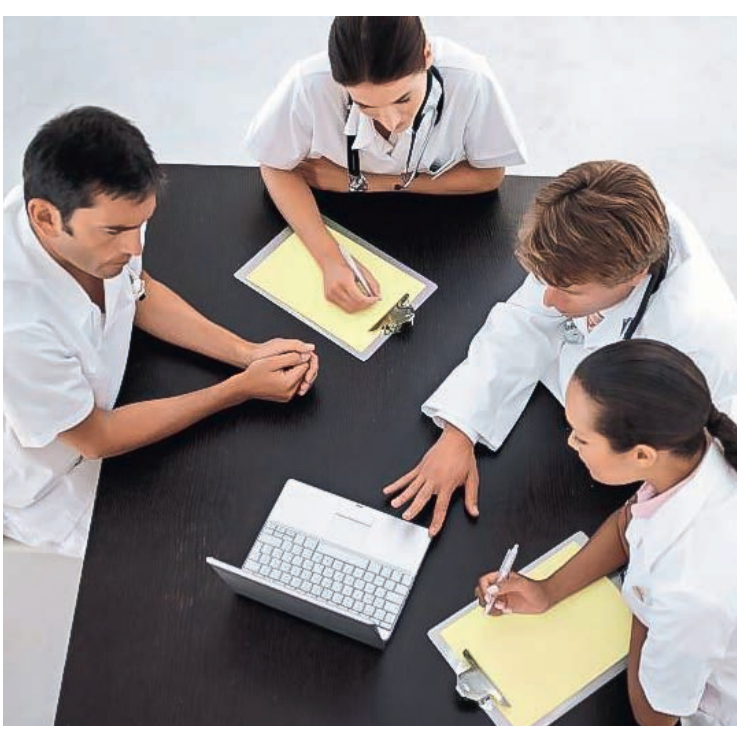

Le concours de I'ISFM s'adresse aux formateurs (personnes individuelles ou équipes) responsables d'établissements de formation postgraduée reconnus en Suisse (image prétexte).

L'une des tâches essentielles de l'Institut suisse pour la formation médicale postgraduée et continue (ISFM) est de soutenir activement les formateurs et de continuer à développer la qualité de la formation médicale postgraduée. Pour remplir sa mission, l'ISFM a donc organisé en 2013 et 2015 un concours visant à promouvoir des projets en faveur de la formation postgraduée.

Forte du succès de ces deux éditions, la direction de l'ISFM a décidé de reconduire le concours.

La diversité, l'originalité et la qualité des projets reçus nous ont fortement impressionnés et le choix des lauréats n'a pas été simple. Forte du succès de ces deux éditions, la direction de l'ISFM a décidé de reconduire le concours afin d'apporter un soutien méthodologique et didactique concret à la formation postgraduée médicale.

Malgré la charge de travail croissante des formateurs et la diminution constante des ressources financières dans le domaine de la santé en Suisse, nous espérons vivement que, cette année encore, vous serez nombreux à nous faire part de vos idées originales et de vos projets novateurs en faveur de la formation médicale.

Les moyens financiers de l'ISFM proviennent des taxes pour l'octroi des titres de spécialiste, c'est-à-dire des médecins en formation postgraduée. Nous attachons donc beaucoup d'importance à employer ces ressources non seulement pour des tâches structurelles et administratives relevant de la formation postgraduée (reconnaissance des programmes et des établissements de formation, évaluation de la formation, octroi des titres), mais aussi et surtout pour contribuer à encourager des idées nouvelles dans ce domaine.

\section{Contenu et méthode libres}

Le concours de l'ISFM s'adresse aux formateurs (personnes individuelles ou équipes) responsables d'établissements de formation postgraduée reconnus en Suisse. Le contenu des projets et la méthode employée sont libres. A titre d'exemple, mentionnons les contenus suivants: moyens auxiliaires didactiques, offres teach the teachers, applications informatiques, outils d'apprentissage interactifs, méthodes de simulation, projets dédiés aux EPA (entrustable professional activities), enseignement des objectifs de formation généraux, etc. Nous souhaitons que cette mise au concours soit aussi large que possible afin de laisser une chance à tous types d'innovations en faveur de la formation.

\section{Les projets soumis doivent préciser les points suivants:}

- Objectif visé

- Description de la méthode, du produit et du résultat visé

- Plus-value pour la formation postgraduée

- Etendue des possibilités d'utilisation et transmissibilité, application dans d'autres institutions

- Critères d'évaluation et de mesure

- Calendrier 
- Budget détaillé du financement global ou partiel

- Coordonnées du responsable de projet

Les projets proposés doivent être réalisables et transposables à d'autres établissements de formation, éventuellement avec des adaptations. Le soutien financier

\section{Un jury composé de membres du comité et de la direction de l'ISFM se chargera de sélectionner les projets.}

alloué peut couvrir l'ensemble du projet ou consister en une contribution à un projet plus important. Les projets soumis doivent pouvoir être achevés d'ici au printemps 2020 et un premier bilan intermédiaire devra être remis à fin 2019.

Vous pouvez envoyer vos projets sous forme électronique d'ici au 15 avril 2019 à siwf[at]fmh.ch avec la mention "Promotion de projets de l'ISFM». Un jury composé de membres du comité et de la direction de l'ISFM se chargera de sélectionner les projets. Le jury aura jusqu'à fin juin pour délibérer. Les contributions de soutien vont de $40000 \mathrm{CHF}$ à $60000 \mathrm{CHF}$ pour le financement de l'ensemble du projet et de $10000 \mathrm{CHF}$ à 20000 CHF pour un financement partiel. La répartition et l'utilisation des moyens financiers à disposition relèvent de la compétence du jury.

Institut suisse pour la formation médicale post-

graduée et continue FMH

Elfenstrasse 18

Case postale 300 CH-3000 Berne 15

Tél. 031359111

siwf[at]fmh.ch le Bulletin des médecins suisses, et présentés lors d'une manifestation organisée par l'ISFM. Les résultats des projets primés seront ensuite mis à la disposition des spécialistes et d'un public plus large afin de les inciter à lancer d'autres projets pour améliorer la qualité de la formation médicale postgraduée.

Crédit photo

Flonline, Francfort

\section{Envoyez vos projets dès maintenant!}

L'ISFM promeut des projets qui soutiennent directement la formation postgraduée des médecins. Avez-vous de nouvelles idées ou disposez-vous d'un concept pertinent que vous souhaiteriez mettre en œuvre?

Alors envoyez votre projet sous forme électronique à siwf[at] fmh.ch avec la mention "Promotion de projets de I'ISFM".

Sont autorisés à participer à ce concours les médecins ayant une fonction de formateur dans un établissement de formation postgraduée suisse reconnu.

Délai de participation: 15 avril 2019

Vous trouverez de plus amples informations sur notre site Internet www.siwf.ch. Pour toute question, veuillez vous adresser à siwf[at]fmh.ch ou au 0313591111.

Mention légale: I'ISFM se réserve le droit, pour des motifs exceptionnels, d'annuler le concours ou d'en modifier les conditions de participation et les subventions octroyées. Toute voie juridique est exclue et aucune correspondance sur le concours ne sera échangée. Les membres du jury n'ont pas le droit de participer au concours. 